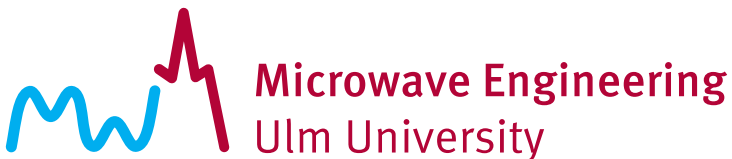

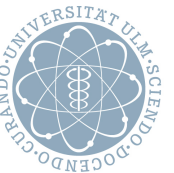

\section{Interference-Robust Processing of OFDM Radar Signals Using Compressed Sensing}

Christina Knill, Benedikt Schweizer, and Christian Waldschmidt

(C) 2020 IEEE. Personal use of this material is permitted. Permission from IEEE must be obtained for all other uses, in any current or future media, including reprinting/republishing this material for advertising or promotional purposes, creating new collective works, for resale or redistribution to servers or lists, or reuse of any copyrighted component of this work in other works. 


\title{
Interference-Robust Processing of OFDM Radar Signals Using Compressed Sensing
}

\author{
Christina Knill ${ }^{1 *}$, Benedikt Schweizer ${ }^{1 *}$, and Christian Waldschmidt ${ }^{1 * *}$ \\ ${ }^{1}$ Institute of Microwave Engineering, Ulm University, 89081 Ulm, Germany \\ * Member, IEEE \\ ** Senior Member, IEEE
}

\begin{abstract}
An essential prerequisite for radars in automotive applications, safety systems, future autonomous driving, and industry applications is the reliable operation even under difficult conditions such as interference from other radars. Recently, digital radar principles such as orthogonal frequency-division multiplexing (OFDM) gained increasing attention. In this letter, an interference-robust processing for OFDM radar signals using compressed sensing (CS) is presented, which is particularly suitable for random OFDM but also applicable to stepped and standard OFDM. It allows robust radar operation in presence of interfering signals that have a comparable low instantaneous bandwidth and high narrowband power. The method is validated experimentally using measurements of an OFDM radar setup at $76 \mathrm{GHz}$ and a state-ofthe-art FMCW radar as an interferer.
\end{abstract}

Index Terms-OFDM, FMCW, interference, radar, automotive, signal processing, compressed sensing, CAMP, signal separation.

\section{INTRODUCTION}

Due to the growing demand for radar-based automotive comfort and safety systems and the limited allowed frequency range for such sensors, the risk of mutual interference increases which in turn can lead to a reduced performance of the sensors [1][2][3]. Nowadays, the majority of radar sensors for automotive applications are based on frequency-modulated continuous waves (FMCW) which typically exhibit a low instantaneous bandwidth. Yet, wideband multicarrier waveforms such as orthogonal frequency-division multiplexing (OFDM) [4] have recently attracted increasing attention in this research area, as they promise more flexibility in waveform design and large unambiguities. However, due to its instantaneous wideband nature, it is likely that an OFDM signal gets disturbed by other sensors in its vicinity. As OFDM is still an emerging new concept in radar science, comprehensive knowledge and research on interference and its treatment is limited so far. In [5], inter- and intra-system interference handling for MIMO OFDM radar networks are investigated where the interfering signal is estimated using pilot symbols and then subtracted from the receive signal. The mutual interference of OFDM radars is investigated in [6] where interfered subcarriers are identified via power detection and removed if necessary. Slow-ramp FMCW disturbance in automotive radar applications is considered in [7] where a frequency domain detection and signal recovery via linear prediction is proposed. In [8] the impact of a fast-chirp FMCW radar on an OFDM radar is shown using measurements. A simple frequency domain detection and mitigation method based on signal leveling is presented.

So far, the procedures have mostly been carried out in advance and detached from signal evaluation. In addition, a multi-stage strategy is usually pursued, in which reliable interference detection is required before signal recovery and signal evaluation. This almost always leads to an increased computational overhead. Therefore, in this letter, an interference-robust joint range-Doppler OFDM processing

Corresponding author: C. Knill (e-mail: christina.knill@uni-ulm.de). Associate Editor: XXX

Digital Object Identifier 10.1109/LSENS.2020.2980165 using compressed sensing (CS) is proposed that does neither require specific interference treatment nor specific detection or mitigation. The proposed approach is inherently robust to interfering signals which are instantaneously narrowband within a wideband OFDM symbol and exhibit comparable high power within an OFDM subcarrier.

CS plays a decisive role in the approach. It has been shown that since radar channels are approximately sparse in range-Doppler and range-azimuth representation by nature ${ }^{1}$, such signals are suitable for CS which offers a powerful tool in radar processing [9]. For OFDM radar, CS is suggested for channel estimation in passive radar application [10], or reconstruction of missing subcarriers [6][11][12]. Furthermore, CS plays an essential role in signal evaluation of random frequency agile OFDM [13][14]. Frequency agile OFDM concepts combine a reduced instantaneous baseband bandwidth with an agile carrier frequency to synthesize a wider band over the signal frame as a major bottleneck for OFDM implementation is the sampling rate limitation caused by the affordable consumer hardware at both transmitter and receiver. The agile carrier frequency can be either chosen deterministically and periodically in a stepped pattern [15] or randomly [14]. Random signal patterns preserve unambiguities and are particularly suitable for the application of CS-based signal evaluation. Therefore, the CS-based interference-robust processing presented in the following will be derived and validated using random OFDM [14], however, it can be straightforwardly transferred to any other OFDM radar concept such as standard and stepped OFDM as well. Moreover, it will be shown that for random OFDM, this approach will not increase the computational cost.

\section{STANDARD AND RANDOM OFDM RADAR}

An OFDM signal frame composed of $M$ symbols of duration $T$ and $N$ orthogonal subcarriers with constant spacing of $\Delta f=1 / T$ has a total signal bandwidth of $W=N \Delta f$. On each subcarrier a sequence of $M$ complex-valued data symbols, that are drawn independently from

${ }^{1}$ A signal is considered (approximately) sparse if it has only few coefficients with significant amplitude in some transformation domain where all other coefficients are (almost) zero. 
a modulation alphabet (e.g., QPSK), is transmitted. At the transmitter, an inverse discrete Fourier transform (IDFT) is performed on the complex data of each OFDM symbol, then the symbols are extended by a cyclic prefix, arranged consecutively, digital-to-analog converted, filtered, and mixed to the desired carrier frequency $f_{c}$.

Based on a standard OFDM frame of size $M \times N$, a corresponding random signal as in [14] is derived by applying two modifications. First, the baseband bandwidth is reduced to $W_{\max }=N_{\max } \Delta f \leq W$ relaxing the requirements to the analog-to-digital converter by a factor $\gamma=W_{\max } / W=N_{\max } / N$ with $0 \leq \gamma \leq 1$ while maintaining the same subcarrier spacing. Second, instead of a fixed carrier frequency, an agile carrier is used that allows the flexible upconversion to any frequency $f_{c}+b \Delta f$ with integer $b \in\left[0, N-N_{\max }-1\right]$. The change of the carrier frequency is initiated randomly after symbol blocks of at most $M_{\max }$ OFDM symbols each. Thereby, a random sampling of the desired equivalent signal frame is imitated. In order to guarantee a sufficient variance of the blocks within the desired bandwidth, which determines the degree of randomness, the length of each block is limited to $M_{\max } \ll M$.

At the receiver the incoming signal is first down-converted to the baseband using the individual carrier frequency of each OFDM symbol and then sampled. Using a discrete Fourier transform (DFT), each OFDM symbol is decomposed into its spectral components, the subcarriers, and the complex modulation is reversed using elementwise complex division. This signal representation is further referred to as frequency domain. For easier depiction, the discrete signal is arranged in a matrix of size $M \times N_{\max }$. Then this matrix is increased to the corresponding actual channel frame size $M \times N$ by shifting each transmitted signal block to its corresponding baseband carrier frequency. Principally, the range-velocity $(r-v)$ evaluation using 2D(I)DFT can then be carried out according to standard OFDM. Since a random signal pattern potentially leads to artifacts in the $r$ - $v$-image, instead a 2D-CS approach comprising a 2D-(I)DFT is used. Similar to [14], a 2D extension of a complex approximate message passing (CAMP) [16] is applied. This approach is called 2D-CAMP in the following.

\section{INTERFERENCE-ROBUST SIGNAL PROCESSING}

Standard OFDM signal evaluation yields a high processing gain and thus a large signal-to-noise ratio (SNR) in the $r$ - $v$-image limited by the inherent OFDM system noise power. However, in presence of high power interference, this performance severely degrades [6][7][8]. In this case, the incoming signal at the receiver is a superposition $\tilde{y}(t)=y(t)+z(t)$ of the desired and the interference signal $z(t)$ within the same frequency band. The corresponding frequency domain frame is

$$
\tilde{\boldsymbol{Y}}=\boldsymbol{Y}+\boldsymbol{Z} \circ \boldsymbol{D}^{*},
$$

where $\boldsymbol{D}^{*}$ is the element wise complex conjugate data matrix, $\boldsymbol{Z}$ encompasses the additional noise and interference, and $\circ$ is the Hadamard product. The corresponding $r$ - $v$-image obtained by 2D(I)DFT then yields

$$
\tilde{\mathcal{I}}=\boldsymbol{W}^{-1} \tilde{\boldsymbol{Y}} \boldsymbol{W}=\boldsymbol{I}+\mathcal{Z}
$$

which is still a superposition of the desired image $\mathcal{I}$ and the interference image $\mathcal{Z}$ where $\boldsymbol{W}$ and $\boldsymbol{W}^{-1}$ are the unitary DFT and IDFT matrices, respectively. The challenge now is to separate the image $\mathcal{I}$ from $\mathcal{Z}$.
In [17] and [18], signal separation based on a variation of the morphological component analysis and CS is used for FMCW and CW interference of FMCW radars, respectively, using the SALSA algorithm. It allows to separate signals that are sparse in two different domains. Sparse means that only few coefficients have a high value while all others are close to zero. However, the task for OFDM is different due to the different signal properties. Moreover, the novel approach should also be able to exploit both range and velocity information, which requires an extension to a $2 \mathrm{D}$ approach. Therefore, an adapted and enhanced signal separation approach called dual-2DCAMP is proposed.

\section{A. Sparse Transformation Domains}

First consider the $r$-v-domain (2). As each target reflection of the OFDM signal yields a peak at the target position in the $r$ - $v$-image, the desired signal is approximately sparse in $\mathcal{I}$. Since this property is independent of waveform and modulation, it is common to all imaging radars and is typicially exploited by CS radar approaches. On the opposite, $\mathcal{Z}$ is not sparse since, for example, the range evaluation $\left(\boldsymbol{Z} \circ \boldsymbol{D}^{*}\right) \boldsymbol{W}$ of the interfering term is a circular convolution of the discrete time OFDM transmit signal and the (sampled) noise signal $z[l]$. However, in general, these signals are independent and decorrelated such that the interference and the noise power get spread approximately equally along the range map which may increases the noise floor. Eventually, the DFT for the velocity estimation preserves this property which means that $\mathcal{Z}$ is not sparse in (2). Therefore, the $r$ - $v$-domain is defined as the sparse domain of the desired signal $\boldsymbol{I}$.

An interfering signal with comparable high instantaneous power but low instantaneous bandwidth, e.g., an FMCW interferer in an OFDM signal, will be compressed into just a few subcarriers $N_{\text {int }} \ll N_{\max } \leq N$ in frequency domain [8] which means that $\boldsymbol{Z}$ in (1) is (block) sparse. Moreover, typically, the interfered samples also exhibit significantly higher amplitudes due to the compression. Since in contrast $\boldsymbol{Y}$ is dense in (1), the frequency domain is defined as the sparse domain for $Z$.

\section{B. Dual-2D-CAMP}

For the separation algorithm, a 2D-CAMP is desired as the basic algorithm. CAMP offers an iterative approximation of the $\ell_{0}$-minimization problem, works without matrix inversion, is selftunable, allows 2D extension, and shows fast convergence. In the basic CAMP, in each iteration $i$, an estimate $X^{[i]}$ of the signal and a residual $\boldsymbol{R}^{[i]}$ are determined, where the latter is also the input to the next iteration.

For the dual-2D extension, two signal estimates $\boldsymbol{X}_{I}^{[i]}$ and $\boldsymbol{X}_{Z}^{[i]}$ are sought. Therefore, in the first step of each iteration, signal separation is performed through two transformations to the sparse domains with halved power using

$$
\begin{aligned}
& \boldsymbol{S}_{I}=\boldsymbol{X}_{I}^{[i-1]}+\frac{1}{2} \boldsymbol{W}^{-1} \boldsymbol{R}^{[i-1]} \boldsymbol{W}, \\
& \boldsymbol{S}_{Z}=\boldsymbol{X}_{Z}^{[i-1]}+\frac{1}{2} \boldsymbol{R}^{[i-1]},
\end{aligned}
$$

where $\boldsymbol{R}^{[0]}=\tilde{\boldsymbol{Y}}$ and $\boldsymbol{X}_{Z}^{[0]}=\boldsymbol{X}_{I}^{[0]}=\mathbf{0}$. Since both $\boldsymbol{R}$ and $\boldsymbol{X}_{Z}$ are frequency domain signals, no transformation is required in (4). Next, in both 


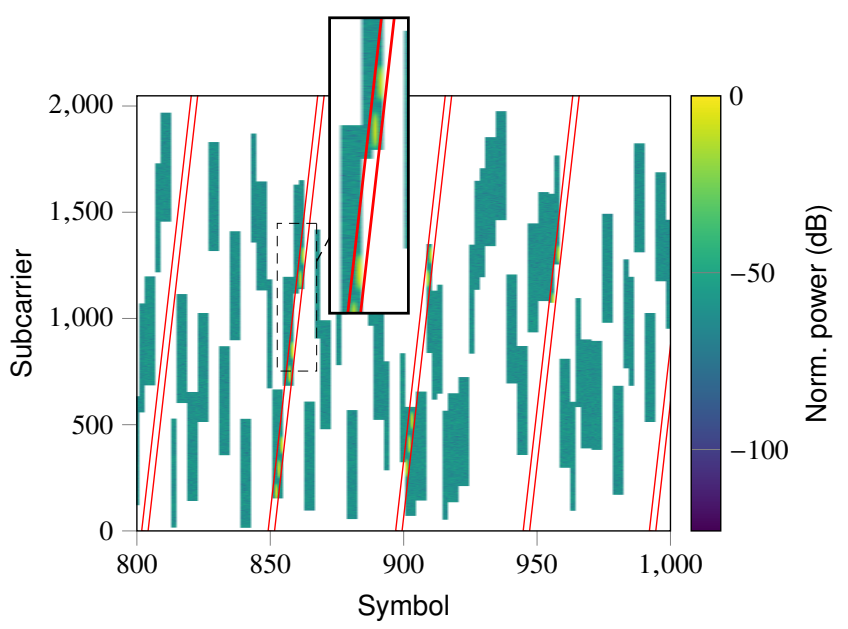

Fig. 1. Excerpts of the frequency domain signal of an FMCW interference in random OFDM with $\gamma=1 / 4\left(W_{\max }=256 \mathrm{MHz}\right)$. The corresponding interfering ramps are framed in red.

domains new estimates $\boldsymbol{X}_{I, Z}^{[i]}$ are determined by soft-thresholding according to

$$
\boldsymbol{X}_{I, Z}^{[i]}=\operatorname{soft}\left(\boldsymbol{S}_{I, Z}, \tau_{I, Z} \cdot \hat{\sigma}_{I, Z}\right)
$$

with thresholds composed of the estimates of the magnitude of the signal samples $\hat{\sigma}_{I, Z}$ multiplied by a scaling factor $\tau_{I, Z}$ similar to [19]. The scaling is independent of $\hat{\sigma}_{I, Z}$ but influences the sensitivity of the threshold step. While a low sensitivity of $\tau_{I}=4.3$ is selected for the OFDM signal to suppress false alarms, a high sensitivity of $\tau_{Z}=\tau_{I} / 3 \approx 1.4$ is selected for the disturbance to ensure complete detection of the interfering signal, accepting the selection of interference-free elements in this case as well.

The decisive step of the algorithm is the determination of a common new residual which will serve as the starting point of the next iteration. For this purpose, the two separated signal estimates must first be transformed back into the common signal domain, where their superposition is used to determine the residual

$$
\boldsymbol{R}^{[i]}=\tilde{\boldsymbol{Y}}-\boldsymbol{P} \circ\left(\boldsymbol{W} \boldsymbol{X}_{I}^{[i]} \boldsymbol{W}^{-1}+\boldsymbol{X}_{Z}^{[i]}\right)+\frac{\left|\boldsymbol{X}_{I}^{[i]}\right|_{0}}{2 N_{\max }} \boldsymbol{R}^{[i-1]} .
$$

$\boldsymbol{P} \in \mathbb{B}^{M \times N}$ is the binary transmission pattern, with $P_{m n}=1$ indicating whether the element was used for transmission, and $|\boldsymbol{x}|_{0}$ denotes the $\ell_{0}$-pseudo-norm, which is the number of nonzero elements of $\boldsymbol{x}$. The output of the algorithm is the desired recovered OFDM signal

$$
\hat{\mathcal{I}}=\boldsymbol{X}_{\mathcal{I}}^{[i]}+\frac{1}{2} \boldsymbol{W}^{-1} \boldsymbol{R}^{[i]} \boldsymbol{W}
$$

and additionally, if desired, an estimate of the interference signal given by

$$
\hat{Z}=\boldsymbol{X}_{Z}^{[i]}+\frac{1}{2} \boldsymbol{R}^{[i]}
$$

\section{Computational Complexity}

Although dual-2D-CAMP can be considered as two intertwined 2D-CAMP algorithms, the complexity is the same. The reason is that the additional signal estimation requires no extra transformation and the threshold determination using a median calculation has only complexity $O(M N)$. Therefore, the complexity of dual-2D-CAMP is determined by the complexity of the 2D-(I)DFT operation of $O(M N \log N)$ which is identical to the complexity of 2D-CAMP.

\section{EVALUATION USING MEASUREMENTS}

The proposed algorithm is validated using interference-free and interfered random OFDM measurements. The radar setup from [15] is used for measurements. The equivalent standard OFDM signal consists of 2048 subcarriers with spacing $500 \mathrm{kHz}$ yielding a total bandwidth of $1.024 \mathrm{GHz}$. The complete OFDM frame consists of 3072 consecutive symbols with duration $2 \mu$ s per symbol. The equivalent isotropically radiated power (EIRP) is $8.5 \mathrm{dBm}$. For the random signal, the instantaneous bandwidth is reduced by a factor $4(\gamma=1 / 4)$, which equals 512 subcarriers and $256 \mathrm{MHz}$. The maximum number of symbols per block is 4 . The interfering state-of-the-art FMCW radar operates at $76 \mathrm{GHz}$ with a bandwidth of $2 \mathrm{GHz}$, an up-ramp time of $89.92 \mu \mathrm{s}$, and a ramp repetition time of $119.92 \mu \mathrm{s}$. Its EIRP is $17 \mathrm{dBm}$.

In Fig. 1, an interfered frequency domain signal is shown. Although interference is present within the equivalent signal frame, the received signal is not necessarily interfered, as it would be the case for standard OFDM. Additionally, the sparseness of $\boldsymbol{Z}$ in (1) is noticeable.

In Figs. 2(a) and (b), the $r$ - $v$-profiles after conventional (random) OFDM processing using 2D-CAMP for measurements with and without interference are shown, respectively. In Fig. 2(c), the $r$ - $v$ image of the interfered measurement after dual-2D-CAMP processing is presented. Additionally, the estimated SNR is given. The observed scene consists of three corner reflectors at ranges of $4.25 \mathrm{~m}, 5.6 \mathrm{~m}$ and $6.1 \mathrm{~m}$ with an interferer at $5.6 \mathrm{~m}$. Comparing the conventionally processed measurements, in case of interference, the SNR severely decreases such that target detection is aggravated and the weakest target at $5.6 \mathrm{~m}$ is barely visible any more. However, if dual-2D-CAMP is used instead, approximately the same SNR as in the interferencefree case is achieved. All targets, even the weak one, become clearly visible again. For better comparison, additionally, the range profiles at $v=0 \mathrm{~m} / \mathrm{s}$ of the interference-free, interfered with 2D-CAMP, and interference-robust dual-2D-CAMP processing is given in Fig. 2(d).

Moreover, in Table 1, the average achieved SNRs in the $r$ - $v$-image for several standard, stepped, and random OFDM measurements are compared for different mitigation approaches i.e. zeroing, leveling, and linear prediction. It shows that the proposed dual-2D-CAMP approach achieves the best results.

It should be mentioned that dual-2D-CAMP can be applied to both interfered and interference-free random, stepped, and standard OFDM radar signals without further adjustments. Moreover, equivalent to the findings of 2D-CS in [14], of course dual-2D-CAMP also works without restrictions for non-stationary target scenes as the kind of range and Doppler information in frequency domain is mathematically the same. Soley their detection and recovery is applied to different dimensions of the frequency domain matrix. As a consequence, the joint $r-v$ processing is independent of the distribution of the sparse coefficients (targets) in the $r$ - $v$-image.

\section{CONCLUSION}

An enhanced processing for OFDM radars is proposed that is robust to interference signals that comprise a comparable low instantaneous bandwidth and high narrowband power, e.g., FMCW signals. It is 


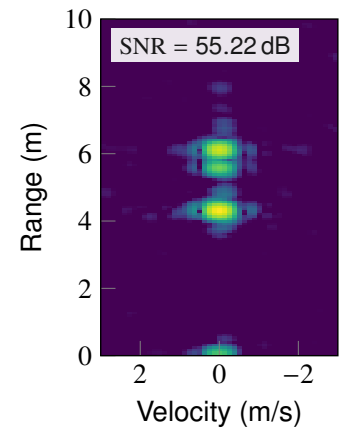

(a)

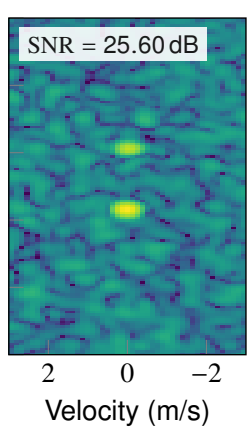

(b)

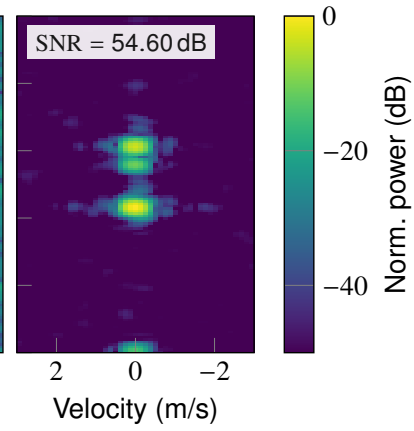

(c)

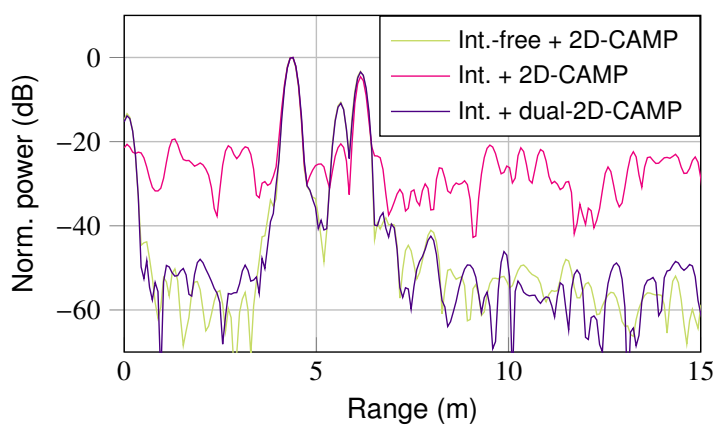

(d)

Fig. 2. In (a)-(c), the $r$-v-images and SNR of random OFDM measurement are shown. In (a) the signal is not interfered and processed using 2D-CAMP, in (b) the signal is interfered and also processed using 2D-CAMP without interference treatment, and in (c) the same interfered signal is processed using dual-2D-CAMP. In (d) a comparison of the range profiles $(v=0 \mathrm{~m} / \mathrm{s})$ of the three results is shown.

TABLE 1. Comparison of average achieved SNRs in the $r$ - $v$-image of different mitigation approaches ( ${ }^{*}$ with 2D-CAMP).

\begin{tabular}{|c|c|c|c|c|}
\hline & & Standard & Stepped $(\gamma=1 / 4)$ & Random $(\gamma=1 / 4)$ \\
\hline & erference-free & $43.9 \mathrm{~dB}$ & $45.0 \mathrm{~dB}$ & $55.1 \mathrm{~dB}^{*}$ \\
\hline & Zeroing & $43.4 \mathrm{~dB}$ & $43.7 \mathrm{~dB}$ & $54.5 \mathrm{~dB}^{*}$ \\
\hline$\frac{\omega}{\omega}$ & Leveling [8] & $43.3 \mathrm{~dB}$ & $43.6 \mathrm{~dB}$ & $53.9 \mathrm{~dB}^{*}$ \\
\hline 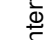 & Linear Pred. [7] & $43.3 \mathrm{~dB}$ & $43.7 \mathrm{~dB}$ & $54.0 \mathrm{~dB}^{*}$ \\
\hline & dual-2D-CAMP & $44.3 \mathrm{~dB}$ & $56.5 \mathrm{~dB}$ & $54.9 \mathrm{~dB}$ \\
\hline
\end{tabular}

shown that through signal separation via dual-2D-CAMP it is possible to separate the interference from the desired OFDM signal during signal evaluation. The approach is particularly suitable for random OFDM but can also be applied for standard or stepped OFDM. Based on random OFDM measurements at $76 \mathrm{GHz}$ with a state-ofthe-art FMCW radar as interferer, the method is validated. With the proposed approach, robust signal evaluation even in the presence of interference is achieved without degradation of target detection performance compared to an interference-free scenario.

\section{ACKNOWLEDGMENT}

This work was supported by the Ministerium für Wissenschaft, Forschung und Kunst (MWK) Baden-Württemberg within the Tech Center a-drive under grant 32-7533-4$10 / 13 / 20$ and the German Bundesministerium für Bildung und Forschung under grant 16EMO0345 (IMIKO-Radar).

\section{REFERENCES}

[1] G. Brooker, "Mutual Interference of Millimeter-Wave Radar Systems," IEEE Trans. Electromagn. Compat., vol. 49, no. 1, pp. 170-181, Feb. 2007.

[2] M. Goppelt, H. L. Blöcher, and W. Menzel, "Automotive radar-investigation of mutual interference mechanisms," Advances in Radio Sci., vol. 8, pp. 55-60, 2010.

[3] T. Schipper, S. Prophet, M. Harter, L. Zwirello, and T. Zwick, "Simulative Prediction of the Interference Potential Between Radars in Common Road Scenarios," IEEE Trans. Electromagn. Compat., vol. 57, no. 3, pp. 322-328, 2015.

[4] N. Levanon, "Multifrequency complementary phase-coded radar signal," IEE Proc. Radar, Sonar and Navigation, vol. 147, no. 6, pp. 276-284, 2000.

[5] Y. L. Sit and T. Zwick, "MIMO OFDM Radar Networks: Inter- \& Intra-System Interference Handling," in Asia-Pacific Microw. Conf., Nov. 2014, pp. 1318-1320.

[6] B. Nuss, Y. L. Sit, and T. Zwick, "A Novel Technique for Interference Mitigation in OFDM Radar Using Compressed Sensing," in IEEE MTT-S Int. Conf. on Microwaves for Intelligent Mobility, Mar. 2017, pp. 143-146.

[7] G. Hakobyan and B. Yang, "A Novel Narrowband Interference Suppression Method for OFDM Radar,' in 24th European Signal Processing Conference (EUSIPCO), no. 2, Aug. 2016, pp. 2230-2234.
[8] C. Knill, B. Schweizer, P. Hügler, and C. Waldschmidt, "Impact of an Automotive Chirp-Sequence Interferer on a Wideband OFDM Radar," European Radar Conference (EuRAD), pp. 8-11, 2018.

[9] M. A. Hadi, S. Alshebeili, K. Jamil, and F. E. A. El-Samie, "Compressive sensing applied to radar systems: an overview," Signal, Image and Video Processing, vol. 9, no. S1, pp. 25-39, Dec. 2015.

[10] C. R. Berger, B. Demissie, J. Heckenbach, P. Willett, and S. Zhou, "Signal Processing for Passive Radar Using OFDM Waveforms," IEEE J. Sel. Topics Signal Process, vol. 4, no. 1, pp. 226-238, Feb. 2010.

[11] G. Hakobyan and B. Yang, "A Novel OFDM-MIMO Radar with Non-equidistant Subcarrier Interleaving and Compressed Sensing," in International Radar Symposium (IRS), 2016.

[12] C. Knill, F. Roos, B. Schweizer, D. Schindler, and C. Waldschmidt, "Random Multiplexing for an MIMO-OFDM Radar With Compressed Sensing-Based Reconstruction," IEEE Microw. Wireless Compon. Lett, vol. 29, no. 4, pp. 300-302, Apr. 2019.

[13] G. Lellouch, R. Pribic, and P. Van Genderen, "Merging Frequency Agile OFDM Waveforms and Compressive Sensing into a Novel Radar Concept," in European Radar Conference (EuRAD), no. export-sep, 2009, pp. 137-140.

[14] C. Knill, B. Schweizer, S. Sparrer, F. Roos, R. F. H. Fischer, and C. Waldschmidt, "High Range and Doppler Resolution by Application of Compressed Sensing Using Low Baseband Bandwidth OFDM Radar," IEEE Trans. Microw. Theory Techn., vol. 66, no. 7, pp. 3535-3546, Jul. 2018.

[15] B. Schweizer, C. Knill, D. Schindler, and C. Waldschmidt, "Stepped-Carrier OFDMRadar Processing Scheme to Retrieve High-Resolution Range-Velocity Profile at Low Sampling Rate," IEEE Trans. Microw. Theory Techn., vol. 66, no. 3, pp. 1610-1618, Mar. 2018.

[16] A. Maleki, L. Anitori, Z. Yang, and R. G. Baraniuk, "Asymptotic Analysis of Complex LASSO via Complex Approximate Message Passing (CAMP)," IEEE Trans. Inf. Theory, vol. 59, no. 7, pp. 4290-4308, Jul. 2013.

[17] F. Uysal and S. Sanka, "Mitigation of Automotive Radar Interference," in IEEE Radar Conference, Apr. 2018, pp. 405-410.

[18] F. Uysal, "Synchronous and Asynchronous Radar Interference Mitigation," IEEE Access, vol. 7, pp. 5846-5852, 2019.

[19] L. Anitori, M. Otten, W. van Rossum, A. Maleki, and R. Baraniuk, "Compressive CFAR Radar Detection,” in IEEE Radar Conference, May 2012, pp. 320-325. 